\title{
Surgical Treatment and Outcome of Patients with De Novo Lung Cancer After Liver Transplantation
}

\author{
FUMIHIRO SHOJI, GOUJI TOYOKAWA, NOBORU HARADA, SHINJI ITOH, \\ NORIFUMI HARIMOTO, TORU IKEGAMI, TATSURO OKAMOTO, \\ YUJI SOEJIMA, TOMOHARU YOSHIZUMI and YOSHIHIKO MAEHARA
}

\author{
Department of Surgery and Science, Graduate School of Medical Sciences, Kyushu University, Fukuoka, Japan
}

\begin{abstract}
Background: De novo malignancy, including primary lung cancer, is one of the limitations to long-term survival after liver transplantation. The purpose of this study was to describe patients who developed de novo lung cancer after living-donor liver transplantation (LDLT) and investigate their clinicopathological features as well as the feasibility of surgical resection. Patients and Methods: We investigated 554 patients who underwent LDLT. Results: De novo lung cancer after LDLT was observed in five (0.9\%) out of 554 studied patients: four men and one woman, aged 6178 years (mean=67 years). All four men had a smoking history. Clinical stages of de novo lung cancer were stage IA in three patients, and stage IB and IV in one patient each. Three out of five patients underwent pulmonary lobectomy and pathological stage was IA in two patients and IIA in one. All patients who underwent surgery stopped immunosuppressive therapy 1 day preoperatively and restarted on postoperative day 1. There were no serious postoperative complications. All three patients are still alive without any recurrence, with survival ranging from 8 to 29 months, with an average of 16.3 months after diagnosis of lung cancer. Conclusion: Although the study population was small, these results suggest that pulmonary lobectomy of de novo lung cancer after LDLT, even under immunosuppressive conditions, is a feasible procedure and may yield a survival benefit.
\end{abstract}

Liver transplantation (LT) is a definite therapy for patients with end-stage liver diseases. Recipients of LT have a good prognosis, thus the number of long-term survivors has

Correspondence to: Fumihiro Shoji, Department of Surgery and Science, Graduate School of Medical Sciences, Kyushu University, 3-1-1, Maidashi, Higashi-ku, Fukuoka, 812-8582, Japan. Tel: +81 926425466, Fax: +81 926425482, e-mail: fshoji@surg2.med.kyushuu.ac.jp

Key Words: De novo lung cancer, living-donor liver transplantation, surgical resection. increased (1). However, the increased incidence of de novo malignancy, including primary lung cancer, is one of the limitations to long-term survival after LT. Lung cancer is the leading cause of cancer-related death worldwide and is the most frequent type of solid organ cancer after LT (2). Surgical treatment for de novo malignancy after LT is an important consideration because an increase in the incidence of cancer in immunocompromised patients has been observed (3). However, the treatment for these diseases is limited by immunosuppressive therapy necessary after LT. Thus, it is necessary to evaluate the treatment of de novo lung cancer, especially surgical resection, after LT. Only a few reports discuss surgery for de novo lung cancer after LT, including postoperative course and surgical outcomes. Therefore, the aim of this study was to describe patients who developed de novo lung cancer after LT and demonstrate the feasibility of surgical resection.

\section{Patients and Methods}

This study was approved by the Ethics Committee of the Kyushu University Hospital, and written informed consent was obtained from all patients. A total of 554 consecutive patients underwent livingdonor LT (LDLT) at Kyushu University Hospital from May 1997 to December 2012. There were 255 men and 299 women, aged 18-74 years (mean $=52$ years). The indications for LDLT were hepatocellular carcinoma (HCC) in 165 patients, liver cirrhosis (LC) in 113, primary biliary cirrhosis (PBC) in 81, fulminant hepatic failure in 54, alcoholic cirrhosis in 21 , primary sclerosing cholangitis in 19 , nonalcoholic steatohepatitis in 16, auto-immune hepatitis in 14, biliary atresia in seven, Wilson's disease in five, and other causes in 59. The indications for LDLT in the Kyushu University Hospital include HCC with neither extrahepatic metastasis nor macroscopic vascular invasion in conventional imaging studies. No limitations were set regarding the size and number of tumours. The pre-transplant workup for HCC consisted of a combination of ultrasonography, dynamic computed tomographic (CT) scans, magnetic resonance imaging, CT angiography and CT during arterioportography, where possible. Patients with extrahepatic metastasis were excluded by head and chest CT scans, bone scintigraphy and positron-emission tomography. Adjuvant chemotherapy and immunosuppressive therapy were previously described (4). 
After LDLT, immunosuppression was initiated. All patients received immunosuppressive therapy with a combination of cyclosporine A (Neoral), mycophenolate mofetil (CellCept), tacrolimus and prednisolone (Predonine). Patients were routinely evaluated as follows. Post-transplant follow-up consisted of abdominal and chest CT, bone scintigraphy and positron-emission tomography imaging studies at 3-month intervals during the first year and yearly thereafter. Tumour markers were checked monthly during the first year and at 3-month intervals thereafter.

\section{Results}

Frequency and clinicopathological features of de novo lung cancer after LDLT. The average follow-up was 56 months (range $=0-207$ months) and $438(79.1 \%)$ patients are currently still living. The actuarial overall 1-, 3-, 5- and 10-year survival rates were $88.1,82.6,79.4$ and $71.9 \%$, respectively (Figure 1).

De novo lung cancer after LDLT was observed in five $(0.9 \%)$ out of 554 patients (Table I): four men and one woman, aged $61-78$ years (mean=67 years). Three of these patients underwent LDLT for HCC and one each for PBC and end-stage hepatitis-C-related LC. Four patients were screened for de novo lung cancer routinely and one because of dyspnoea. The four men had a history of smoking. The average interval between LDLT and diagnosis of de novo lung cancer was 78 months (range=0-162 months). Immunosuppressive drugs immediately before treatment of de novo lung cancer were as follows: tacrolimus in two patients and cyclosporine A with/without mycophenolate mofetil in three patients. Clinical stage of de novo lung cancer was IA in three patients and IB and IV in one patient each.

Treatment of patients with de novo lung cancer after LDLT. Treatment of the five patients with de novo lung cancer is shown in Table II. Primary tumours were located in the left upper lobe in two patients and right lower lobe in three. Pathological type of de novo lung cancer was adenocarcinoma and squamous cell carcinoma in two patients each, and unknown in the other patient. Three patients underwent surgical resection. Contraindications for surgery were as follows. Patient 1 had adenocarcinoma with malignant effusion and multiple pulmonary metastases; patient 2 had both clinical stage IB non-small cell lung cancer and unresectable advanced maxillary cancer. Therefore, patient 1 received best supportive care and patient 2 radiotherapy.

Patients undergoing pulmonary resection after LDLT. Table III shows the surgical outcome of the three patients who underwent pulmonary resection. The average tumour size was $14.7 \mathrm{~mm}$ (range=13-17 mm). Patients 4 and 5 underwent right lower lobectomy and patient 3 left upper lobectomy. Patient 3 underwent pulmonary resection 3 months after LDLT,

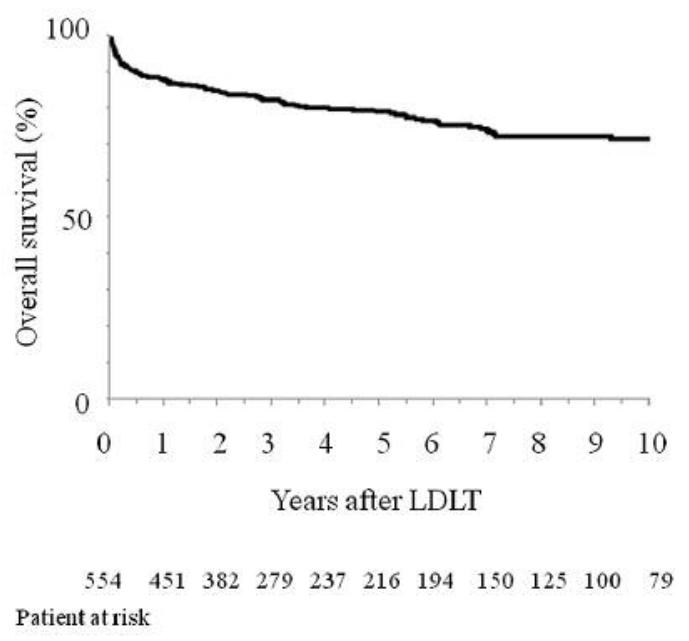

Figure 1. Overall survival curve for patients receiving living donor liver transplantation $(L D L T)$.

because de novo lung cancer was synchronously discovered with LC. The average operating time was 270 minutes (range=236-313 minutes) and the average amount of blood loss was $82 \mathrm{~g}$ (range $=0-140 \mathrm{~g}$ ). The postoperative course of the three patients is also shown in Table III. All the patients stopped immunosuppressive therapy 1 day preoperatively and restarted on postoperative day (POD)1. In all patients, the thoracic tube was removed within 5 POD (average=3.6 POD, range $=2-5$ POD). There were no serious complications and the average hospital stay was 9.7 days (range=7-15 days).

Outcome of patients with de novo lung cancer after LDLT. Treatment and outcome of patients with de novo lung cancer after LDLT are shown in Table IV. De novo lung cancer was pathologically confirmed as stage IA in two patients and IIA in one patient. All three patients that underwent surgical resection are still alive, although the other two patients that were unable to undergo surgery died. The survival range for the patients who underwent surgical resection was 29-174 months (mean=122 months) after LDLT. In contrast, the survival range for the patients who we unable to undergo surgical resection was 33-57 months (mean=45 months) after LDLT. In addition, the survival range for the patients who underwent surgical resection was 8-29 months (mean=16 months) after diagnosis of de novo lung cancer.

\section{Discussion}

There is an increased risk of de novo cancer in patients after LT because post-transplant survival has dramatically improved. De novo malignancy after LT remains the leading cause of late death, significantly impairing long-term survival 
Table I. Clinical features of patients with de novo lung cancer after living-donor liver transplantation (LDLT).

\begin{tabular}{lccccccc}
\hline $\begin{array}{l}\text { Patient } \\
\text { no. }\end{array}$ & Gender & $\begin{array}{c}\text { Age } \\
\text { (years) }\end{array}$ & $\begin{array}{c}\text { Reason for } \\
\text { screening }\end{array}$ & $\begin{array}{c}\text { Smoking } \\
\text { status }\end{array}$ & $\begin{array}{c}\text { Liver } \\
\text { disease }\end{array}$ & Immunosuppression & $\begin{array}{c}\text { Interval between LDLT and } \\
\text { diagnosis of de novo lung cancer }\end{array}$ \\
\hline 1 & Male & 67 & Dyspnoea & Former & PBC & Tacrolimus & 33 \\
2 & Male & 65 & RS & Former & HCC & Cyclosporine A & 39 \\
3 & Female & 67 & RS & Non & LC & Cyclosporine A+mycophenolate mofetil & 0 \\
4 & Male & 61 & RS & Former & HCC & Tacrolimus & 162 \\
5 & Male & 78 & RS & Former & HCC & Cyclosporine A & 156 \\
\hline
\end{tabular}

HCC: Hepatocellular carcinoma; LC: liver cirrhosis (end-stage hepatitis C-related); PBC: primary biliary cirrhosis; RS: routine screening.

Table II. Clinicopathological features and treatment of patients with de novo lung cancer after living-donor liver transplantation.

\begin{tabular}{lcccc}
\hline Patient no. & Primary site & Pathological type & Clinical stage & Treatment \\
\hline 1 & RLL & Adenocarcinoma & IV & Best supportive care \\
2 & LUL & Unknown & IB & Radiotherapy \\
3 & LUL & Adenocarcinoma & IA & Surgery \\
4 & RLL & Squamous cell carcinoma & Surgery \\
5 & RLL & Squamous cell carcinoma & IA & Surgery \\
\hline
\end{tabular}

HCC: Hepatocellular carcinoma; LUL: left upper lobe; RLL: right lower lobe.

(2). The risk of de novo cancer increases with age and time after LT. Desai et al. showed that the standardised incidence ratio for all cancers after LT was 2- to 3-fold compared to the general population adjusted for age, gender and year (5).

The most common site for developing de novo solid organ malignancies after LT is the lungs (2). It was shown that 824 out of $89,036(0.925 \%)$ LT patients developed de novo lung cancer (2). Recent small studies have also revealed that the incidence rate of primary lung cancer after LT is $0.3-0.8 \%$ (8-10). The frequency (five out of 544 patients, $0.9 \%$ ) of $d e$ novo lung cancer in the current study was similar to that in previous studies.

Previous studies have shown that the time to diagnosis of de novo lung cancer was 47-71 months after LT (6-8). Moreover, Zhou et al. showed that cancer risk was increased at 1 year after LT, gradually increasing and reaching a peak at 6-10 years of follow-up, and that during the first 6 months and $>16$ years after LT, cancer risk decreased (2). Our mean time to diagnosis of 78 months is similar to that in previous studies.

Smoking is a risk factor of de novo lung cancer after LT $(13,14)$. Mangus et al. reported that the incidence of de novo cancer was higher for patients with, compared to those without a history of smoking (15). In the present study, all four male patients also had a history of smoking.

The major cause of the development of de novo malignancy is related to the loss of immunovigilance induced by immunosuppressive drugs (16). The risk of de novo
Table III. Surgical treatment of patients with de novo lung cancer after living-donor liver transplantation. All patients discontinued immunosuppressive drug use one day prior to surgery and recommenced it one day after surgery.

\begin{tabular}{lccc}
\hline $\begin{array}{l}\text { Patient } \\
\text { no. }\end{array}$ & $\begin{array}{c}\text { Surgical } \\
\text { procedure }\end{array}$ & $\begin{array}{c}\text { Chest drain } \\
\text { removal (POD) }\end{array}$ & $\begin{array}{c}\text { Hospital } \\
\text { stay (days) }\end{array}$ \\
\hline 3 & LUL & 5 & 15 \\
4 & RLL & 2 & 7 \\
5 & RLL & 4 & 10 \\
\hline
\end{tabular}

LUL: Left upper lobectomy; RLL: right lower lobectomy, POD: postoperative day.

cancer can be reduced by simple lifestyle changes, including smoking cessation, as well as maintaining minimum exposure to immunosuppression (17). Thus, LT patients with a history of smoking might benefit from careful post-LT surveillance for de novo cancer, although our small study cannot lead to any such definitive conclusion.

In the present study, de novo lung cancer was stage IA in three patients and IB and IV in one patient each. Stage I de novo lung cancer in these patients was detected by routine screening, which therefore detects post-LT cancer at an early stage, leading to surgical resection and improved survival. Unfortunately, patient 2 was found to have unresectable advanced maxillary cancer at the same time (double de novo cancer). 
Table IV. Outcome of patients with de novo lung cancer after living-donor liver transplantation (LDLT).

\begin{tabular}{lcccc}
\hline $\begin{array}{l}\text { Patient } \\
\text { no. }\end{array}$ & $\begin{array}{c}\text { Pathological } \\
\text { stage }\end{array}$ & $\begin{array}{c}\text { Survival after } \\
\text { LDLT (months) }\end{array}$ & $\begin{array}{c}\text { Survival after diagnosis of } \\
\text { de novo lung cancer (months) }\end{array}$ & Status \\
\hline 1 & - & 33 & 0 & Dead \\
2 & - & 57 & 29 & Dead \\
3 & IA & 29 & 12 & Alive \\
4 & IIA & 174 & 8 & Alive \\
5 & IA & 163 & Alive \\
\hline
\end{tabular}

There is no definite consensus regarding timing for discontinuation or restarting immunosuppressive drugs in LDLT patients undergoing surgery. In the present study, all patients undergoing surgery stopped their immunosuppressive therapy 1 day preoperatively and restarted on POD1, as previously reported (9). Consequently, there were no serious postoperative complications. All three patients are still alive and survival range was 6-29 months (mean=15.7 months) after diagnosis of lung cancer. The other two patients died from $d e$ novo cancer.

We previously reported the results of surgical resection of pulmonary malignant tumours, including recurrence and de novo malignancy, such as primary lung cancer or mucosa-associated lymphoid tissue lymphoma, after LDLT (9). However, we did not experience any operable cases of de novo lung cancer in that study. In addition, none of patients with tumour recurrence underwent pulmonary lobectomy, only limited surgery such as wedge resection. Only a few studies have reported surgical resection of $d e$ novo lung cancer after LT (10. 11). Jiménez et al. reported that one patient with stage IIIB primary lung cancer received exploratory thoracotomy and one with stage IA primary lung cancer underwent a curative operation (10). Another study reported pulmonary wedge resection in a patient with stage IA pulmonary adenocarcinoma (11). Therefore, it is unclear whether major lung resection, including lobectomy, which is considered the standard procedure for primary lung cancer, would be feasible for LDLT patients. We previously reported a synchronous case with fatal LC and lung cancer (12). This case was successfully treated for LC by LDLT and pulmonary lobectomy for primary lung cancer was performed 3 months after LDLT. Moreover, we newly experienced two operable cases of de novo lung cancer after LDLT. As a result, we have seen five patients with de novo lung cancer, including three who underwent pulmonary lobectomy after LDLT. Thus, pulmonary lobectomy for de novo lung cancer after LDLT might be a safe and promising therapy in LDLT patients under immunosuppressive conditions.

In conclusion, although we included a small number of patients, we reported the frequency and surgical results of $d e$ novo lung cancer after LDLT. These results suggest that pulmonary lobectomy of de novo lung cancer after LDLT is a safe and acceptable procedure and may benefit patients receiving LDLT.

\section{Conflicts of Interest}

None declared.

\section{References}

1 Soejima Y, Shirabe K, Taketomi A, Yoshizumi T, Uchiyama H, Ikegami T, Ninomiya M, Harada N, Ijichi $\mathrm{H}$ and Maehara $\mathrm{Y}$ : Left lobe living donor liver transplantation in adults. Am J Transplant 12: 1877-1885, 2012.

2 Zhou J, Hu Z, Zhang Q, Li Z, Xiang J, Yan S, Wu J, Zhang M and Zheng S: Spectrum of de novo cancers and predictors in liver transplantation: analysis of the scientific registry of transplant recipients database. PLoS One 11: e0155179, 2016.

3 Murray KF and Carithers RL Jr.: ASLD practice guidelines: evaluation of the patient for liver transplantation. Hepatology 41 : 1407-1432, 2005.

4 Soejima Y, Taketomi A, Yoshizumi T, Uchiyama H, Aishima S, Terashi T, Shimada M and Maehara Y: Extended indication for living liver donor transplantation in patients with hepatocellular carcinoma. Transplantation 83: 893-899, 2007.

5 Desai $\mathrm{R}$ and Neuberger J: Donor transmitted and de novo cancer after liver transplantation. World J Gastroenterol 20: 6170-6179, 2014.

6 Sanchez EQ, Marubashi S, Jung G, Levy MF, Goldstein RM, Molmenti EP, Fasola CG, Gonwa TA, Jennings LW, Brooks BK and Klintmalm GB: De novo tumors after liver transplantation: a single-institutional experience. Liver Transpl 8: 285-291, 2002.

7 Peyrègne $\mathrm{V}$, Ducerf $\mathrm{C}$, Adham $\mathrm{M}$, de la Roche $\mathrm{E}$, Berthoux $\mathrm{N}$, Bancel B, Bizollon $\mathrm{T}$ and Baulieux J: De novo cancer after orthotopic liver transplantation. Transplant Proc 30: 1484-1485, 1998.

8 Aseni P, Vertemati M, De Carlis L, Sansalone CV, Bonacina E, Minola E, Oreste $\mathrm{P}$, Vizzotto L and Rondinara G: De novo cancers and post-transplant lymphoproliferative disorder in adult liver transplantation. Pathol Int 56: 712-715, 2006.

9 Shoji F, Kawano D, Ikegami T, Soejima Y, Taketomi A, Yano T and Maehara Y: Surgical resection of pulmonary malignant tumors after living-donor liver transplantation. Ann Thorac Surg. 88: 206-211, 2009. 
10 Jiménez C, Manrique A, Marqués E, Ortega P, Loinaz C, Gómez R, Meneu JC, Abradelo M, Moreno A, López A and Moreno E: Incidence and risk factors for the development of lung tumors after liver transplantation. Transpl Int 20: 57-63, 2007.

11 Kanamoto M, Imura S, Morine Y, Ikemoto T, Mori H, Arakawa Y, Hanaoka J, Tokunaga T, Sugimoto K, Nishi M and Shimada M: De novo lung cancer diagnosed 32 months after liver transplantation: report of a case. Surg Today 41: 1280-1283, 2011.

12 Taniguchi D, Harimoto N, Takeishi K, Itoh S, Yamashita Y, Ikegami T, Yoshizumi T, Kawanaka H, Shirabe K, Morodomi Y, Tagawa T, Okamoto $\mathrm{T}$ and Maehara Y: Liver transplantation followed by pulmonary resection complicated with end-stage liver cirrhosis: a case report. Anticancer Res 35: 3411-3414, 2015.

13 de Perrot M, Wigle DA, Pierre AF, Tsao MS, Waddell TK, Todd TR and Keshavjee SH: Bronchogenic carcinoma after solid organ transplantation. Ann Thorac Surg 75: 367-371, 2003.

14 Jiménez-Romero $\mathrm{C}$ and Justo-Alonso I, Cambra-Molero F, Calvo-Pulido J, García-Sesma Á, Abradelo-Usera M, CasoMaestro $\mathrm{O}$ and Manrique-Municio A: Incidence, risk factors and outcome of de novo tumors in liver transplant recipients focusing on alcoholic cirrhosis. World J Hepatol 7: 942-953, 2015.
15 Mangus RS, Fridell JA, Kubal CA, Loeffler AL, Krause AA, Bell JA, Tiwari S and Tector J: Worse long-term patient survival and higher cancer rates in liver transplant recipients with a history of smoking. Transplantation 99: 1862-1868, 2015.

16 Finkenstedt A, Graziadei IW, Oberaigner W, Hilbe W, Nachbaur K, Mark W, Margreiter R and Vogel W: Extensive surveillance promotes early diagnosis and improved survival of de novo malignancies in liver transplant recipients. Am J Transplant 9: 2355-2361, 2009.

17 Neuberger J: An update on liver transplantation: a critical review. J Autoimmun 66: 51-59, 2016.

Received March 1, 2017

Revised March 21, 2017

Accepted March 24, 2017 